\title{
Who tweets about sports law?
}

\author{
Johan Lindholm ${ }^{1}$
}

Published online: 17 October 2019

( ) T.M.C. Asser Instituut 2019

\section{Introduction}

Social media is ubiquitous. ${ }^{1}$ It is difficult to fathom that there was no Facebook fifteen years ago and that social media's spread to seemingly every aspect of society has essentially happened in the last decade. This includes professional life as, for example, evidenced by LinkedIn's more than 650 million users. Not even the academic debate, which traditionally prides itself with being deliberate, nuanced, and comparatively slow, has escaped social media. While scholars vary regarding to what extent they use and engage with social media, studies have shown that researchers generally find social media stressful, risky, of poor quality, and at odds with conducting high-quality research. ${ }^{2}$ There is nevertheless evidence that more and more scholarly communication takes place on social media, ${ }^{3}$ including on research-dedicated platforms such as ResearchGate and Academia.edu. ${ }^{4}$

The overwhelming majority of users' interaction with social media can be sorted into one of two categories: producing and consuming content. This also holds true for professional use of social media, including in the context of academia. Researchers produce social media content that includes describing their research findings, spreading scientific news, and more generally sharing their thoughts, ideas, and reactions. Social media can be used to attract more attention to papers ${ }^{5}$ and in this sense functions as a "networked loudspeaker," 6 a tool with more and less positive uses. ${ }^{7}$ Scholars also act as professional social media consumers using social media to stay up to date with the latest development in their fields. However, because social media is social interaction and easily and reliably collectable, researchers have for some time also used social media as a tool for studying social interaction. ${ }^{8}$ This short contribution is a humble example of this.

Over time, the use of social media in a professional capacity has spread to the field of sports law where actors

Johan Lindholm

johan.lindholm@umu.se

1 Department of Law, Umeå University, 90187 Umeå, Sweden

interested in and working with sports law produce and consume content and (hopefully) communicate with each other. One very popular platform for this is Twitter, ${ }^{9}$ and one can in this regard speak of a "sports law Twittersphere."10 There is little doubt that social media's impact on the world is real and extensive. ${ }^{11}$ Much like Twitter as a whole constitutes "a globally distributed public conversation" that influences which phenomena are discussed and how they are understood, ${ }^{12}$ the sports law Twittersphere influences the global sports law discussion and affects what the participants focus on and how they think about it. This editorial seeks to explore who are the actors that shape the sports law Twittersphere.

In order to answer this question, I have collected and studied 2,127 tweets about sports law: All original tweets ${ }^{13}$ posted over a period of 71 days ${ }^{14}$ that contain the hashtag "\#sportslaw," 15 thereby targeting the particular "hashtag

\footnotetext{
${ }^{1}$ Kaplan and Haenlein (2010, p. 61) define social media as "a group of Internet-based applications that build on the ideological and technological foundations of Web 2.0, and that allow the creation and exchange of User Generated Content."

2 Sugimoto et al. (2017, p. 2038) and sources cited.

${ }^{3}$ Ibid.

4 van Noorden (2014).

5 Ortega (2016).

${ }^{6}$ Duval (2018).

7 See Roelofs and Gallien (2017).

${ }^{8}$ See, e.g., Bakshy et al. (2011), Wu et al. (2011).

${ }^{9}$ It should in this context be acknowledged that Twitter is not equally popular in all countries and that many of the countries where it is used are Western and English speaking. See Leetaru et al. (2013), Hawelka et al. (2014).

${ }^{10}$ Cf. Bruns et al. (2014), Bruns and Enli (2018).

11 See, e.g., Castells (2015).

12 Bruns and Enli (2018), pp. 129-130.

13 That is, all retweets have been removed from the dataset.

${ }^{14}$ Between 20 July and 29 September 2019.

15 It is highly unlikely that the data include "false positive," i.e., that a tweet with the hashtag does not address a topic that most would consider falling within the field of sports law. Obviously, many sports law-related tweets do not contain a hashtag identifying them as such
} 


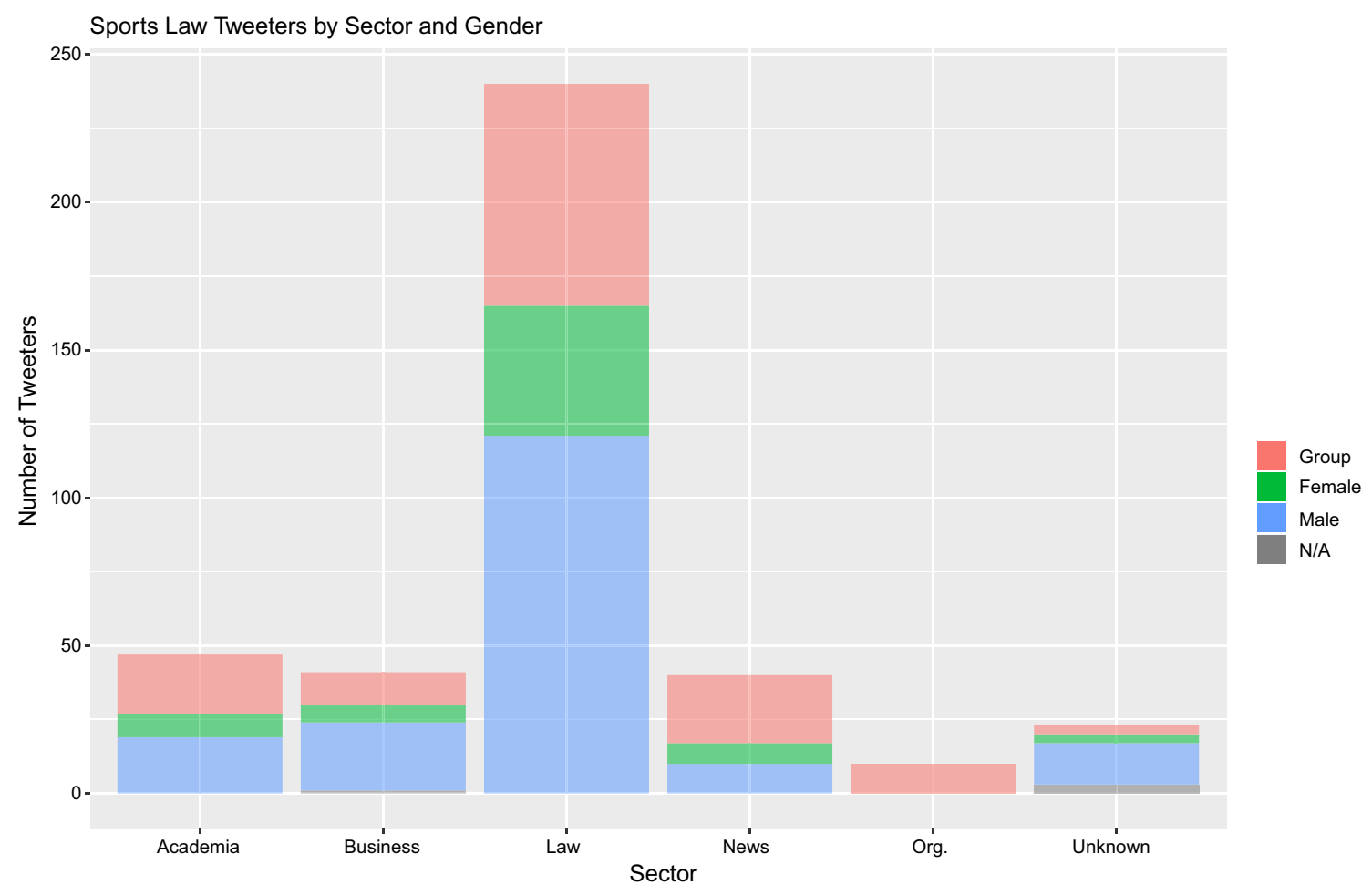

Fig. 1 Sports law tweeters by sector and gender

public" 16 or "legal sub-field"17 of interest. These tweets were posted by 401 unique users, a group that are here referred to as "sports law tweeters." Some observations can be made with regard to the characteristics of these sports law tweeters. ${ }^{18}$

First, many users of Twitter might primarily think of Twitter accounts as personal accounts. One of the defining characteristics of social media is its ability to connect

\section{Footnote 15 (continued)}

and are therefore not included in the data. However, adding other hashtags or search terms (e.g., "CAS" or "doping + law OR legal OR court") would inevitably bias this study toward a particular, preconceived notion of sports law and risk including false positives. There are also no reasons to believe that the hashtag would be more used by particular tweeters or for particular sports-law-related topics and thereby bias the study. While this approach biases the study toward English-language tweets, and consequently English-speaking users, it should also be acknowledged that English is the language most commonly on the Internet in general and on Twitter. Without disrespecting the importance of other major languages, English is bound to be the dominant language of a global discussion about sports law.

16 Bruns and Enli (2018), p. 130.

17 Duval (2018).

18 See Fig. 1. Sports Law Tweeters by Sector and Gender. These characteristics were coded primarily based on information provided on Twitter but also other social media (e.g., LinkedIn) and employer Web sites. individuals across the globe (human connectedness). ${ }^{19}$ However, more than $35 \%$ of all sports law Twitter accounts are institutional accounts managed by a collective, most commonly law firms specialized in sports law, ${ }^{20}$ but also news organizations ${ }^{21}$ and universities. ${ }^{22}$ This is a quite significant portion, perhaps greater than expected. These accounts are operated by individuals who produce the content. It is nevertheless worth considering who the organization tweeting is and what interests it has in participating in the Twittersphere. ${ }^{23}$

Second, as one might have expected, men dominate the sports law Twittersphere, outnumbering female sports law tweeters almost four to one with only marginal differences between different sectors. ${ }^{24}$ There is obviously room for significant improvement. However, in evaluating the situation, one should bear in mind that men are more than twice as

\footnotetext{
${ }^{19}$ Cf. van Dijck and Poell (2013), pp. 8-9.

2075 accounts or circa $19 \%$ of all sports law tweeters.

2123 accounts or circa $6 \%$.

2220 accounts or circa $5 \%$.

${ }^{23}$ Cf. Duval (2018) (discussing institutional tweeting in legal research).

${ }^{24}$ Of all 258 personal sports law Twitter accounts where the gender of the holder could be determined, 68 or ca $26 \%$ had a female user.
} 


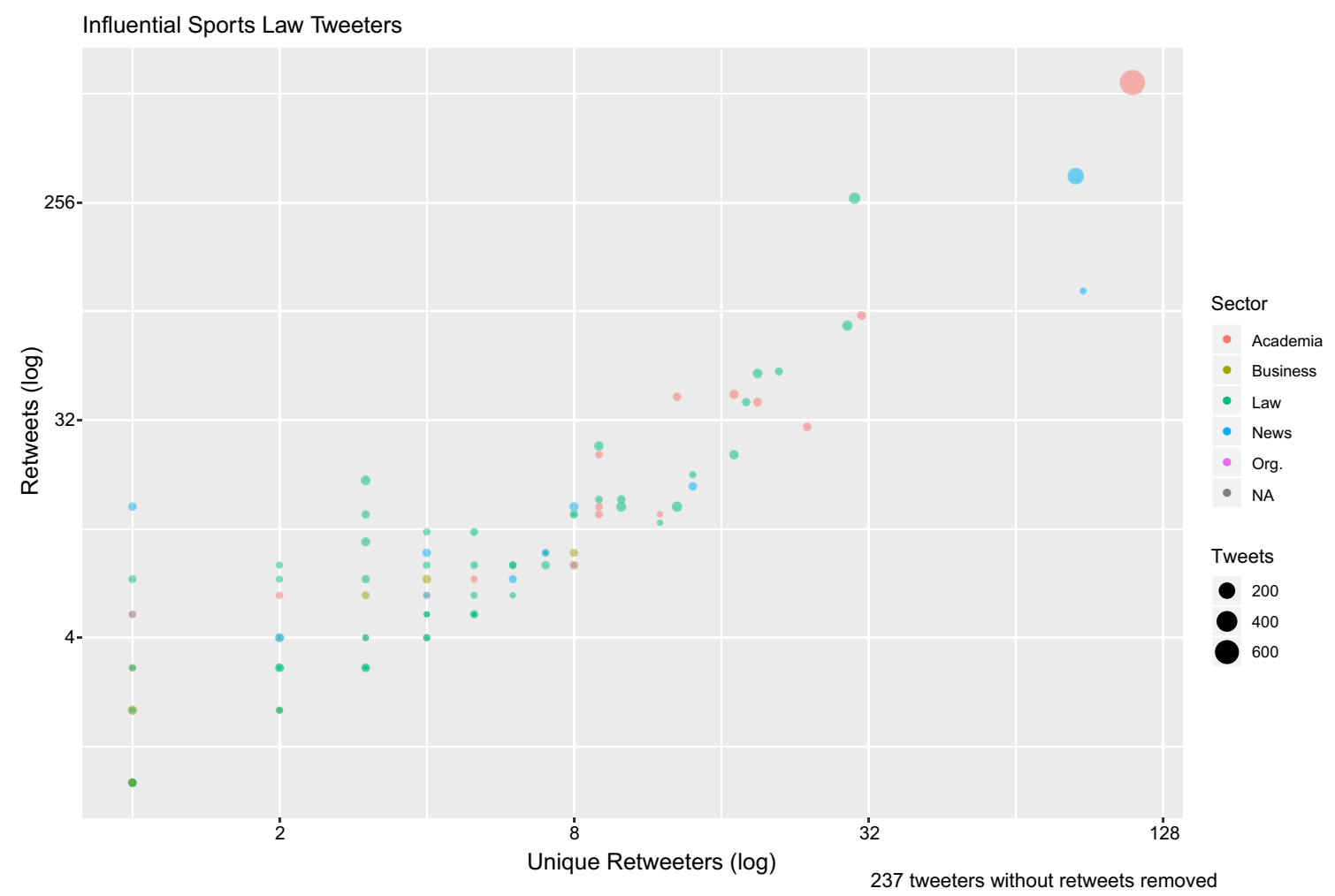

Fig. 2 Influential sports law tweeters

common as women among Twitter users, ${ }^{25}$ and that there is a well-recognized gender gap in sport interest. With that in mind, one would perhaps have expected the gender imbalance to be even starker.

Third, also largely unsurprisingly, $60 \%$ of the sports law tweeters are legal professionals, either individual lawyers or law firms operating a collective account. Most of the remaining accounts belong to individual or collective actors operating in the fields of (i) academia, including universities, student organizations, and individual academics, (ii) news/ media, including individual journalists as well as various types of media outlets ( $\mathrm{tv}$, radio, online, etc.) targeting the general public, lawyers, sports lawyers, or the sports sector more broadly, and (iii) the sports business more broadly, that is professional actors that serve the sports sector, excluding traditional lawyer services, such as agents, accountants, and consultants. Tweeters in these sectors each account for roughly $10 \%$ of all sports law tweeters. Finally, a smaller number of accounts are held by nonprofit organizations such

\footnotetext{
25 Statista, Distribution of Twitter users worldwide as of July 2019, by gender. https://www.statista.com/statistics/828092/distribution-ofusers-on-twitter-worldwide-gender/. Visited 30 September 2019.
}

as anti-doping think tanks, lawyers' associations, and legal research groups. ${ }^{26}$

This description has treated the sports law Twittersphere as an arena where all participants are equally important. This is decidedly not the case. Compared to mass media, there are very few costs associated with or other barriers to producing social media content. However, in reality - and in contrast to the egalitarian ethos-a few users command much of the attention and exercise great influence on social media. ${ }^{27}$

This is also true for the sports law Twittersphere. There is no universally accepted measurement of influence on social media in general or Twitter in particular. ${ }^{28}$ There are, however, good arguments for using retweets as a proxy for influence. ${ }^{29}$ When a Twitter user retweets a tweet, he or she indicates an interest in the original author's opinion. It also indicates the original tweeter's power to communicate his/her/its opinions beyond his/her/its immediate

\footnotetext{
26 Tweeters that did not fit any of these classes or lacked necessary information were excluded.

27 See, e.g., Huffaker (2010), Åkerlund (2019).

${ }^{28}$ See, e.g., Cha et al. (2010), Kwak et al. (2010), Weng et al. (2010).

29 Tweets are generally retweeted shortly after posted. However, to ensure that tweets have reached "peak retweet," a minimum of $24 \mathrm{~h}$ and average of $74 \mathrm{~h}$ passed between a sports law tweet were posted and the number of retweets measured along with the retweeting users' usernames.
} 
network. ${ }^{30}$ Retweets are here studied in two different ways: (i) the total number of times a sports law tweeter's sports law tweets have been retweeted ("number of retweets") and (ii) the number of unique users ${ }^{31}$ that have retweeted a sports law tweeter's sports law tweets ("number of unique retweeters"). ${ }^{32}$

These measurements show that influence within the sports law Twittersphere is as inequitable distributed as in social media more generally. ${ }^{33}$ The vast majority of all sports law tweeters have no or extremely little (measurable) influence on other users. On the other side of the scale, a very small number of sports law tweeters command most of the (measurable) influence. Even though it is universally true across social media, it may come as a surprise that the sports law Twittersphere has a one-percenter-type situation: The most retweeted user has received more retweets than the least retweeted half.

There seems to be several different successful approaches for becoming an influential sports law tweeter. While some high-influence tweeters are also high-frequency tweeters, there is no absolute correlation between volume and influence: Some of the most influential tweeters post relatively few tweets, and conversely, there are relatively frequent tweeters with limited influence. There are also no clear differences between tweeters based on the sector to which they belong. More surprisingly, there is no strong correlation between the number of followers and influence as measured here. This suggests that there is a skill to writing influential tweets, ${ }^{34}$ but could also indicate that are Twitter users with many followers who sometimes tweet about sports law but whose following is largely interested in other issues.

\section{References}

Åkerlund M (2019) The importance of influential actors' platform usage patterns in (re)producing Swedish far-right discourse on Twitter. Forthcoming publication

Bakshy E, Hofman J, Mason W, Watts D (2011) Identifying 'Influencers' on Twitter. In: WSDM 2011-Proceedings of the fourth ACM international conference on Web search and data mining, pp 65-74

Bruns A, Enli G (2018) The Norwegian Twittersphere: structure and dynamics. Nordicom Review 39(1):129-148

\footnotetext{
30 Åkerlund 2019 and sources cited.

31 This includes users outside what is here referred to as sports law tweeters.

32 By using both measurements, one can, e.g., catch users with a small but very active following. However, as seen in Fig. 2, in the data, they largely follow each other. The number of followers was considered as a possible alternative or additional metric, but, as addressed below, it is problematic for measuring influence within a particular polity.

${ }^{33}$ See Fig. 2. Influential Sports Law Tweeters. Please note the double logarithmic scale.

${ }^{34}$ For some advice, see Duval (2018).
}

Bruns A, Burgess J, Highfield T (2014) A 'Big Data' approach to mapping the Australian Twittersphere. In: Arthur PL, Bode K (eds) Advancing digital humanities: research, methods, theories. Palgrave Macmillan, Houndmills, pp 113-129

Castells M (2015) Networks of outrage and hope-social movement in the internet age, 2nd edn. Polity, Cambridge)

Cha M, Haddadi H, Benevenuto F, Gummad KP (2010) Measuring user influence on twitter: the million follower fallacy. In 4th international AAAI conference on weblogs and social media, pp 10-17

Duval A (2018) Publish tweets and blogs or perish legal academia in times of social media. Tilburg Law Review 23:91

Hawelka B, Sitko I, Beinat E, Sobolevsky S, Kazakopoulos P, Ratti C (2014) Geo-located Twitter as proxy for global mobility patterns. Cartogr Geogr Inf Sci 41(3):260-271

Huffaker D (2010) Dimensions of leadership and social influence in online communities. Human Commun Res 36(4):593-617

Kaplan AM, Haenlein M (2010) Users of the world, unite! The challenges and opportunities of Social Media. Bus Horiz 53:59-68

Kwak H, Lee C, Park H, Moon S (2010) What is twitter, a social network or a news media? In: Proceedings of the 19th international conference on World Wide Web, pp 591-600

Leetaru K, Wang S, Cao G, Padmanabhan A, Shook E (2013) Mapping the global Twitter heartbeat: the geography of Twitter. First Monday 18(5). https://doi.org/10.5210/fm.v18i5.4366. https://first monday.org/ojs/index.php/fm/article/view/4366/3654. Accessed 13 Aug 2019

Ortega JL (2016) To be or not to be on Twitter, and its relationship with the tweeting and citation of research papers. Scientometrics 109:1353-1364

Roelofs P, Gallien M (2017) Clickbait and impact: how academia has been hacked. https://blogs.1se.ac.uk/impactofsocialscienc es/2017/09/19/clickbait-and-impact-how-academia-has-beenhacked/. Accessed 30 Sept 2019

Sugimoto CR, Work S, Larivière V, Haustein S (2017) Scholarly use of social media and altmetrics: a review of the literature. J Assoc Inf Sci Technol 68(9):2037-2062

van Dijck J, Poell T (2013) Understanding Social Media Logic. Media Commun 1(1):2-14

van Noorden R (2014) Scientists and the social network. Nature 512:126-129

Weng J, Lim E, Jiang J, He Q (2010) Twitterrank: finding topic-sensitive influential Twitterers. In: Proceedings of the third ACM international conference on Web search and data mining, pp 261-270

Wu S, Hofman J, MasonW, Watts D (2011) Who says what to whom on twitter. In: Proceedings of the third ACM international conference on web search \& data mining, pp 705-714

Publisher's Note Springer Nature remains neutral with regard to jurisdictional claims in published maps and institutional affiliations. 